\title{
Identification of the prognostic value of lymphocyte-to-monocyte ratio in patients with HBV-associated advanced hepatocellular carcinoma
}

\author{
YING-FEN HONG $^{1 *}$, ZHAN-HONG CHEN $^{1,2^{*}}$, LI WEI $^{1 *}, \mathrm{XIAO}-K U N ~ M A^{1}, \mathrm{XING} \mathrm{LI}^{1}$, \\ JING-YUN WEN ${ }^{1}$, TIAN-TIAN WANG ${ }^{1}$, XIU-RONG CAI ${ }^{1}$, DONG-HAO WU ${ }^{1}$, JIE CHEN $^{1}$, \\ DAN-YUN RUAN ${ }^{1}$, ZE-XIAO LIN $^{1}$, QU LIN ${ }^{1}$, MIN DONG $^{1}$ and XIANG-YUAN WU ${ }^{1}$ \\ ${ }^{1}$ Department of Medical Oncology and Guangdong Key Laboratory of Liver Disease, \\ The Third Affiliated Hospital of Sun Yat-sen University, Guangzhou, Guangdong 510630; \\ ${ }^{2}$ Department of Medical Oncology of Sun Yat-sen University Cancer Center, State Key Laboratory of Oncology in \\ South China, Collaborative Innovation Center for Cancer Medicine, Guangzhou, Guangdong 510060, P.R. China
}

Received May 12,2016; Accepted April 13, 2017

DOI: $10.3892 / 01.2017 .6420$

\begin{abstract}
The inflammatory microenvironment serves an important function in the progression of hepatocellular carcinoma (HCC). Peripheral blood lymphocyte-to-monocyte ratio (LMR), as a novel inflammatory biomarker combining an estimate of host immune homeostasis with the tumor microenvironment, has been identified to be a predictor of clinical outcomes in a number of malignancies. The present study aimed at investigating the prognostic value of LMR in patients with hepatitis B virus (HBV)-associated advanced HCC. A total of 174 patients with HBV-associated advanced HCC, without fever or signs of infections, were analyzed. Clinicopathological parameters, including LMR, were evaluated to identify predictors of overall survival time. Univariate and multivariate analysis was performed using Cox's proportional hazards model. A threshold value was determined using a time-dependent receiver operating characteristic curve. Univariate and multivariate analysis identified LMR as an independent prognostic factor in overall survival (OS) time in patients with $\mathrm{HBV}$-associated advanced HCC $(\mathrm{P}<0.05)$. The threshold value of LMR was 2.22. All
\end{abstract}

Correspondence to: Dr Xiang-Yuan Wu or Dr Zhan-Hong Chen, Department of Medical Oncology and Guangdong Key Laboratory of Liver Disease, The Third Affiliated Hospital of Sun Yat-sen University, 600 Tianhe Road, Guangzhou, Guangdong 510630, P.R. China

E-mail: wuxiangy@mail.sysu.edu.cn

E-mail: sumszhanhong@163.com

*Contributed equally

Key words: lymphocyte-to-monocyte ratio, inflammation, chronic hepatitis B virus infection, prognosis, advanced hepatocellular carcinoma patients were divided into either a low LMR group $(\leq 2.22)$ or a high LMR group (>2.22). The OS time of the high LMR group was significantly longer compared with the low LMR group $(\mathrm{P}<0.001)$. Patients in the high LMR group exhibited a significantly increased 3-month and 6-month OS rate, compared with that of the patients within the low LMR group $(\mathrm{P}<0.001)$. An increased level of LMR was significantly associated with the presence of metastasis, ascites and increased tumor size $(\mathrm{P}<0.01)$. LMR is an independent prognostic factor of HBV-associated advanced HCC patients and an increased baseline LMR level indicates an improved prognosis.

\section{Introduction}

Hepatocellular carcinoma (HCC) ranks fifth among the primary causes of cancer-associated mortality in males and ninth in females (1). In China, HCC is the most common type of cancer and the highest cause of cancer mortality in males $<60$ years (2), due to widespread hepatitis B virus (HBV) infection which almost $90 \%$ of patients with HCC experience. Hepatitis $\mathrm{C}$ virus (HCV) infection, alcoholism and obesity are additional risk factors for HCC in the western world (3). At present, the only treatment for $\mathrm{HCC}$ is hepatic resection which is restricted to the patients who have localized lesions arising in non-cirrhotic livers, or in cirrhotic livers with well-preserved hepatic function (4). However, the majority of patients are initially diagnosed with advanced HCC and therefore the majority of patients with HCC are unable to be treated by resection. Despite substantial progress achieved in the treatment for advanced HCC in previous years, including targeted therapy (sorafenib, a kinase inhibitor drug) and interventional therapy, the overall 5-year survival rate remains unsatisfactory. The prognosis and treatment of HCC depends on the tumor stage, the patient's physical status and liver function $(4,5)$. Clinicopathological factors including microvascular invasion, novel extrahepatic lesions and portal vein tumor thrombus (PVTT) have been used to predict survival time in patients with advanced $\operatorname{HCC}(6,7)$. However, these 
aforementioned clinical tumor parameters may only partially explain the prognostic heterogeneity of HCC.

The inflammatory microenvironment serves an important function in the development and progression of HCC. In China, chronic liver inflammation primarily contributes to the development of HCC (8). In addition, the host response in systemic inflammation has been considered to be an independent prognostic factor for patients with HCC (9-12). C-reactive protein $(13,14)$, white blood cell counts, platelet counts $(15)$, neutrophil counts (16) and monocyte counts (17) have been primarily investigated as clinical indicators of inflammation for cancer. Previous studies have indicated that the development of HCC is associated with alterations of peripheral blood white blood cells. The principal alterations include neutrophilia with relative lymphocy topenia or thrombocytosis. White blood cells, along with the cytokines and chemokines secreted, serve a role in the viability, maturation and differentiation of cells within the tumor microenvironment. Peripheral blood lymphocyte-to-monocyte ratio (LMR), an indication of lymphocytopenia and an increased monocyte count, has been identified to be a novel inflammatory biomarker. LMR combines an estimate of host immune homeostasis and tumor microenvironment and has been identified as a predictor for clinical outcomes in a variety of malignancies, including breast (18), renal (19,20), lung (21) and colorectal (22) cancer. Recently, a study elucidated that LMR was an independent prognostic factor in patients with HBV-associated HCC following curative resection (23). However, to the best of our knowledge, there have been no studies regarding the prognostic value of LMR in advanced HCC until the present study, which aimed at clarifying the prognostic value of LMR in patients with $\mathrm{HBV}$-associated advanced HCC.

\section{Materials and methods}

Patients. The Clinical Ethics Review Board at The Third Affiliated Hospital of Sun Yat-sen University (Guangdong, China) approved the present study, and written informed consent was obtained from the patients or their family members. Medical records of 174 consecutive Chinese patients with HBV-associated advanced HCC presented to The Third Affiliated Hospital of Sun Yat-sen University (Guangdong, China) between September 2008 and June 2010, were reviewed and retrospectively analyzed in the present study. The inclusion criteria of the patients were as follows: i) Patients were diagnosed with advanced HCC. The diagnosis of HCC was confirmed using pathological analysis or the patients met the radiological criteria from The American Association for the Study of Liver Diseases (4), including computed tomography (CT) and magnetic resonance imaging (MRI). Patients with advanced HCC exhibited stage $\mathrm{C}$ or D, according to The Barcelona Clinic Liver Cancer Staging for Hepatocellular Carcinoma (BCLC) (24). ii) All the patients had chronic HBV infection and were negative for anti-HCV antibody. iii) Antiviral therapy with oral nucleos(t)ide analogues was recommended for all patients, although the patients did not receive antitumor treatment recommended by the National Comprehensive Cancer Network guidelines (25) including chemotherapy, targeted therapy or locoregional therapies (percutaneous ethanol injection or transarterial
Table I. Baseline demographics of all patients included in the present study.

\begin{tabular}{|c|c|}
\hline Characteristic & Patients $(\mathrm{n}=174)$ \\
\hline \multicolumn{2}{|l|}{ Sex } \\
\hline Male & $90.2 \%(157 / 174)$ \\
\hline Female & $9.8 \%(17 / 174)$ \\
\hline Age, years & $55(19-85)$ \\
\hline Ascites & $66.1 \%(115 / 174)$ \\
\hline Encephalopathy & $2.9 \%(5 / 174)$ \\
\hline Karnofsky performance status & $60(20-90)$ \\
\hline \multicolumn{2}{|l|}{ Laboratory parameters } \\
\hline Leukocytes, $\times 10^{9}$ cells/ 1 & $6.84(1.84-25.09)$ \\
\hline Neutrophils, $\mathrm{x} 10^{9}$ cells/1 & $4.89(1.11-22.44)$ \\
\hline ALC, $x 10^{9}$ cells $/ 1$ & $1.23(0.32-4.18)$ \\
\hline $\mathrm{AMC}, \mathrm{x} 10^{9}$ cells $/ 1$ & $0.60(0.08-3.85)$ \\
\hline Hemoglobin, g/l & $115.3(56.0-177.0)$ \\
\hline Platelets, $\times 10^{9}$ cells $/ 1$ & $156.3(4.0-503.0)$ \\
\hline LMR & $2.57(0.39-7.0)$ \\
\hline$\leq 2.22$ & $49.4 \%(86 / 174)$ \\
\hline$>2.22$ & $50.6 \%(88 / 174)$ \\
\hline 3-Month mortality rate, $\%$ & $59.2 \%(103 / 174)$ \\
\hline 6-Month mortality rate, $\%$ & $74.1 \%(129 / 174)$ \\
\hline \multicolumn{2}{|l|}{ Child-Pugh grade } \\
\hline 0 & $20.7 \%(36 / 174)$ \\
\hline 1 & $44.8 \%(78 / 174)$ \\
\hline 2 & $34.5 \%(60 / 174)$ \\
\hline \multicolumn{2}{|l|}{ TNM stage } \\
\hline I & $9.8 \%(17 / 174)$ \\
\hline II & $11.5 \%(20 / 174)$ \\
\hline III & $56.3 \%(98 / 174)$ \\
\hline IV & $22.4 \%(39 / 174)$ \\
\hline Lymph node metastasis & $30.5 \%(53 / 174)$ \\
\hline Distant metastases & $20.7 \%(36 / 174)$ \\
\hline Portal vein thrombosis & $72.4 \%(126 / 174)$ \\
\hline \multicolumn{2}{|l|}{ NCCN-TNM } \\
\hline $\mathrm{T} 1$ & $10.9 \%(19 / 174)$ \\
\hline $\mathrm{T} 2$ & $13.8 \%(24 / 174)$ \\
\hline T3-T4 & $75.3 \%(131 / 174)$ \\
\hline
\end{tabular}

ALC, absolute lymphocyte count; AMC, absolute monocyte count; LMR, lymphocyte-to-monocyte ratio; NCCN, National Comprehensive Cancer Network; TNM, tumor-node-metastasis.

chemoembolization). The exclusion criteria of the patients were as follows: i) Patients exhibited a fever or additional manifestations of acute infections; ii) patients exhibited previous or secondary cancers and coexistent hematological disorders; and iii) patients did not possess information required for the present study or lost follow-up within 6 months.

Collection of patient information. Acquisition of patient results from electronic charts was approved by the institutional ethics committee of the Third Affiliated Hospital of Sun Yat-sen 
Table II. Independent prognostic factors for overall survival in patients with hepatitis B virus-associated advanced hepatocellular carcinoma according to univariate and multivariate analysis.

\begin{tabular}{|c|c|c|c|c|c|c|}
\hline \multirow[b]{2}{*}{ Variables } & \multicolumn{3}{|c|}{ Univariate analysis } & \multicolumn{3}{|c|}{ Multivariate analysis } \\
\hline & HR & $95 \% \mathrm{CI}$ & P-value & HR & $95 \% \mathrm{CI}$ & P-value \\
\hline Age & 0.983 & $0.971-0.996$ & 0.009 & & & \\
\hline Leukocytes, $\mathrm{x} 10^{9}$ cells/1 & 1.085 & $1.042-1.130$ & 0.000 & & & \\
\hline Neutrophils, $\times 10^{9}$ cells/l & 1.130 & $1.077-1.186$ & 0.000 & 1.104 & $1.046-1.165$ & 0.000 \\
\hline AMC, $\times 10^{9}$ cells $/ 1$ & 1.348 & $1.013-1.794$ & 0.040 & & & \\
\hline ALC, $\times 10^{9}$ cells $/ 1$ & 0.832 & $0.613-1.129$ & 0.237 & & & \\
\hline LMR & 0.765 & $0.675-0.867$ & 0.000 & 0.858 & $0.754-0.976$ & 0.020 \\
\hline Platelets, $\mathrm{x} 10^{9}$ cells/ 1 & 1.002 & $1.001-1.004$ & 0.006 & & & \\
\hline AST, U/1 & 1.001 & $1.000-1.001$ & 0.000 & & & \\
\hline AFP, ng/dl, $>400$ vs. $\leq 400$ & 2.30 & $1.649-2.309$ & 0.000 & 1.739 & $1.221-2.478$ & 0.002 \\
\hline Ascites, yes vs. no & 2.05 & $1.444-2.909$ & 0.000 & & & \\
\hline Tumor number, $<3$ vs. $\geq 3$ & 1.306 & $1.106-1.542$ & 0.002 & & & \\
\hline Tumor size, $\mathrm{cm},<1$ vs. $\geq 1$ & 3.450 & $1.682-7.077$ & 0.001 & & & \\
\hline PVTT, yes vs. no & 2.661 & $1.792-3.951$ & 0.000 & 1.984 & $1.296-3.037$ & 0.002 \\
\hline
\end{tabular}

HR, hazard ratio; CI, confidence interval; ALC, absolute lymphocyte count; AMC, absolute monocyte count; LMR, lymphocyte-to-monocyte ratio; AST, aspartate transaminase; ALP, alkaline phosphatase; AFP, $\alpha$-fetoprotein; PVTT, portal vein tumor thrombus.

University (Guangdong, China). Clinical examination, laboratory studies and imaging studies (CT or MRI) were required for baseline evaluation. Information was collected at the time of the initial diagnosis of advanced HCC. The overall survival (OS) time was calculated between the date of diagnosis and the date of mortality or last contact, for surviving patients.

Demographic information, laboratory results [including routine blood tests, liver function parameters, serum $\alpha$-fetoprotein (AFP) level], BCLC stage and Karnofsky performance status (26) were retrospectively analyzed from the patients' clinical records. Tumor-associated variables, including maximal tumor diameter, number of tumor nodules and PVTT were obtained from medical image diagnostic results. The peripheral blood absolute lymphocyte count (ALC) and absolute monocyte count (AMC) were derived from the complete blood cell count prior to initiation of treatment, with the LMR calculated by dividing the lymphocyte count by the monocyte count.

Statistical analysis. Fisher's exact test (two-tailed) or $\chi^{2}$ test was used to analyze categorical variables, whereas Student's t-test or non-parametric tests were used to analyze continuous variables. Using OS time as the end-point, the optimal threshold value of LMR using receiver operating curve (ROC) analysis was obtained when the Youden index (sensitivity + specificity-1) (27) was maximal. Subsequently, patients with an LMR greater than the threshold value were classified as high LMR (HLMR) and the remaining patients were classified as low LMR (LLMR). Survival curves for the two groups were plotted using the Kaplan-Meier estimator method and the significance was analyzed using the log-rank test. Significant factors for OS time in Cox's univariate analysis were assessed using Cox's proportional hazards model (forward method) to determine the independent prognostic predictors. Associations between LMR levels and baseline characteristics, including clinicopathological features, were analyzed using $\chi^{2}$ test. All statistical analysis was conducted using SPSS (version 20.0; IBM Corp., Armonk, NY, USA) and Stata software (version 12.0; StataCorp LP, College Station, TX, USA). For all tests, $\mathrm{P}<0.05$ was considered to indicate a statistically significant difference.

\section{Results}

Patient characteristics. In the present study, of the 174 patients with HBV-associated advanced HCC, 157 (90.2\%) were male and the mean age was 55 years (range, 19-85 years). The mean counts of white blood cells, neutrophils, ALC, AMC and platelets were $6.84 \times 10^{9}$ cells/1 [range, (1.84-25.09) $\mathrm{x} 10^{9}$ cells/1], $4.89 \times 10^{9}$ cells $/ 1$ [range, $(1.11-22.44) \times 10^{9}$ cells $/ 1$, $1.23 \times 10^{9}$ cells $/ 1$ [range, $(0.32-4.18) \times 10^{9}$ cells $\left./ 1\right], 0.60 \times 10^{9}$ cells $/ 1$ [range, (0.08-3.85)x $10^{9}$ cells/1] and 156.3 cells/1 [range, (4-503) $\mathrm{x} 10^{9}$ cells/1], respectively. The median LMR was 2.57 (range, $0.39-7.0)$. A total of $53(30.5 \%)$ patients with lymph node metastasis and $36(20.7 \%)$ patients with initial distant metastasis were recorded from newly diagnosed patients. According to BCLC staging criteria, the number of patients with stage $\mathrm{C}$ and D advanced HCC was 95 (54.6\%) and 79 (45.4\%), respectively. Of the patients included in the present study, $152(87.4 \%)$ patients succumbed to cancer-associated disease. The 3-month and 6-month mortality rates were 59.2 and $74.1 \%$, respectively. Additional information for the patients' baseline demographics are presented in Table I.

Independent prognostic factors for overall survival. To identify the optimal peripheral blood inflammatory and immune biomarker for patient prognosis, the impact of leukocyte, neutrophil, ALC, AMC and LMR on survival outcomes was investigated. In the univariate analysis, leukocytes, 
neutrophils, AMC and LMR were identified to be significant prognostic factors, with $\mathrm{P}<0.001$ for leukocytes, neutrophils and LMR, $\mathrm{P}=0.04$ for $\mathrm{AMC}$ and no significant difference indicated for ALC. Additional independent prognostic factors that may be associated with overall survival time included age, platelets, aspartate transaminase (AST), AFP, ascites, tumor number, tumor size and PVTT $(\mathrm{P}<0.05)$. The aforementioned factors identifying statistical significance were assessed using Cox's multivariate regression analysis to determine the significance of independent factors. In the multivariate analysis, LMR [hazard ratio (HR), 0.858; 95\% confidence interval (CI), 0.754-0.976; $\mathrm{P}=0.020]$, neutrophil (HR, 1.104; 95\% CI, 1.046-1.165; P<0.001), PVTT (HR, 1.984; 95\% CI, 1.296-3.037; $\mathrm{P}<0.001)$ and AFP $>400 \mathrm{~g} / \mathrm{ml}(\mathrm{HR}, 1.739$; 95\% CI, 1.221-2.478; $\mathrm{P}=0.002)$ remained independent prognostic predictors (Table II).

Optimal threshold value for LMR. LMR was used as the test variable and the OS time was employed as the state variable for LMR. According to the ROC curve, the optimal threshold value for LMR was 2.22 (sensitivity, 63.1\%; specificity, 69.0\%) (Fig. 1). Patients were dichotomized into either the HLMR group or LLMR group, on the basis of the optimal threshold value. A total of 86 patients $(49.4 \%)$ were included in the LLMR group $(\leq 2.22)$ and 88 patients $(50.5 \%)$ were included in the HLMR group (>2.22).

Comparison of patients between the HLMR and LLMR groups. To determine the association between LMR and clinicopathological features of patients with $\mathrm{HBV}$-associated advanced HCC, comparisons between the HLMR group and LLMR group were made (Table III). The results indicated that HLMR group exhibited a tendency to present distant metastasis, ascites and a larger tumor size $(\mathrm{P}<0.01)$. The LLMR level was significantly associated with increased leukocytes, neutrophils, AMC, ALC, AST, total bilirubin, alkaline phosphatase $(\mathrm{P}<0.001)$ and albumin $(\mathrm{P}=0.045)$. In the survival analysis, the 3 -month and 6-month survival rates of the HLMR group were significantly increased compared with those of the LLMR group $(\mathrm{P}<0.001)$. A Kaplan-Meier estimator curve identified that the OS rate was significantly decreased in the LLMR group compared with that of the HLMR group ( $\mathrm{P}<0.001$, Fig. 2).

\section{Discussion}

HCC has been established as life-threatening disease and it has been reported that majority of patients are initially diagnosed with advanced HCC. Despite the efforts of clinicians to prolong the survival time of patients with advanced HCC, effective therapies are limited. A number of clinicopathological characteristics, including tumor size and thrombosis, have become the primary determinants of existing therapies and predictors of prognosis of patients with HCC. However, these aforementioned factors are far from meeting medical needs.

In 1863, Virchow (28) first proposed the association between inflammation and cancer. Subsequently, a number of studies have indicated the important function of inflammation in early carcinogenesis, development, metastasis and therapeutic response of cancers (29-31). HCC was a typical example of inflammation-associated cancer as the majority

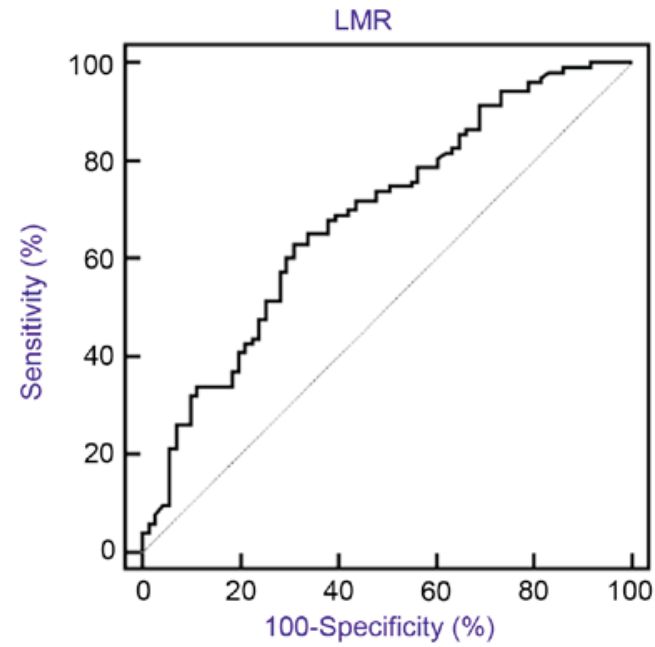

Figure 1. ROC curve assessing the threshold value of LMR for survival analysis in patients. The optimal threshold value of LMR was 2.22, determined using ROC curves, and the area under curve was 0.686 (95\% confidence interval, $0.611-0.754 ; \mathrm{P}<0.001)$ with a sensitivity and specificity of 63.1 and $69.0 \%$, respectively. ROC, receiver operating characteristic; LMR, lymphocyte-to-monocyte ratio.

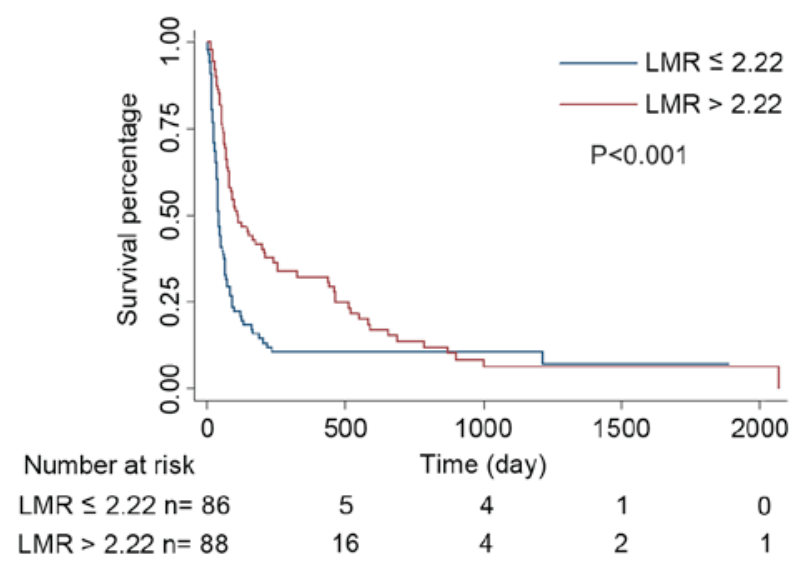

Figure 2. Overall survival curves of 174 patients with hepatitis B virus-associated advanced hepatocellular carcinoma. Kaplan-Meier estimator survival curves demonstrated that patients with LMR $\leq 2.22$ had decreased overall survival rates, compared with those with LMR $>2.22$ ( $\mathrm{P}<0.001, \log$-rank). LMR, lymphocyte-to-monocyte ratio.

of HCC developed in the setting of chronic liver damage and inflammation, particularly in China. Therefore, in the present study, to illustrate the function of LMR in HBV-associated advanced $\mathrm{HCC}$, only patients with HBV infection were included. Pretreatment numbers of peripheral blood cells, including neutrophils, ALC, AMC and platelets, have been suggested to be marked prognostic factors in a number of types of malignancies. Neutrophils, as the largest proportion of leukocytes in human peripheral blood, secrete mediators including cytokines and matrix metalloproteinase- 9 to react to cancer $(32,33)$ and alter their phenotype, similar to granulocytic myeloid-derived suppressor cells, allowing them to exhibit distinct characteristics of maturity, tumor cytotoxicity and immune suppression (34). During tumor development, the inflammatory response may cause lymphocytopenia, leading to the inhibition of suppression of tumor progression (35). 
Table III. Clinical features between the high and low LMR groups.

\begin{tabular}{|c|c|c|c|}
\hline \multirow[b]{2}{*}{ Characteristics } & \multicolumn{2}{|c|}{ LMR } & \multirow[b]{2}{*}{ P-value } \\
\hline & $\leq 2.22(\mathrm{n}=86)$ & $>2.22(n=88)$ & \\
\hline Age, years & $54.4 \pm 4.51$ & $56.8 \pm 13.2$ & 0.217 \\
\hline \multicolumn{4}{|l|}{ Sex } \\
\hline Male & 76 & 81 & \multirow[t]{2}{*}{0.415} \\
\hline Female & 10 & 7 & \\
\hline Leukocytes, $\mathrm{x} 10^{9}$ cells/ 1 & $8.0 \pm 4.3$ & $5.7 \pm 2.1$ & 0.000 \\
\hline Neutrophils, $x 10^{9}$ cells/l & $6.1 \pm 3.5$ & $3.7 \pm 1.8$ & 0.000 \\
\hline Monocytes, $x 10^{9}$ cells/ 1 & $0.8 \pm 0.6$ & $0.4 \pm 0.2$ & 0.000 \\
\hline Lymphocytes, $\times 10^{9}$ cells $/ 1$ & $1.1 \pm 0.6$ & $1.4 \pm 0.5$ & 0.000 \\
\hline Platelets, $\times 10^{9}$ cells/ 1 & $164.1 \pm 109.4$ & $148.7 \pm 95.0$ & 0.584 \\
\hline Hemoglobin, g/l & $112.3 \pm 21.7$ & $118.3 \pm 21.7$ & 0.068 \\
\hline AST, U/l & $266.4 \pm 323.9$ & $164.6 \pm 200.8$ & 0.002 \\
\hline Total bilirubin, $\mu \mathrm{mol} / \mathrm{l}$ & $105.2 \pm 136.7$ & $72.6 \pm 108.0$ & 0.002 \\
\hline Albumin, $\mathrm{g} / \mathrm{l}$ & $32.0 \pm 6.3$ & $33.9 \pm 6.1$ & 0.045 \\
\hline ALP, U/1 & $212.7 \pm 144.2$ & $148.3 \pm 76.1$ & 0.006 \\
\hline BUN, mmol/l & $6.8 \pm 4.2$ & $6.1 \pm 3.2$ & 0.546 \\
\hline Creatinine, $\mu \mathrm{mol} / 1$ & $75.2 \pm 30.2$ & $76.4 \pm 27.6$ & 0.137 \\
\hline AFU, U/1 & $30.3 \pm 17.4$ & $29.7 \pm 18.5$ & 0.758 \\
\hline GGT, U/1 & $292.9 \pm 268.4$ & $215.3 \pm 179.7$ & 0.091 \\
\hline Prothrombin time, sec & $70.6 \pm 18.1$ & $75.4 \pm 19.5$ & 0.088 \\
\hline INR & $1.3 \pm 0.3$ & $1.3 \pm 0.3$ & 0.125 \\
\hline \multicolumn{4}{|l|}{ Lymph node metastasis } \\
\hline Absent & 54 & 67 & \multirow[t]{2}{*}{0.056} \\
\hline Present & 32 & 21 & \\
\hline \multicolumn{4}{|l|}{ Distant metastasis } \\
\hline Absent & 60 & 78 & \multirow[t]{2}{*}{0.002} \\
\hline Present & 36 & 0 & \\
\hline \multicolumn{4}{|l|}{ PVTT } \\
\hline Absent & 18 & 30 & \multirow[t]{2}{*}{0.052} \\
\hline Present & 68 & 58 & \\
\hline \multicolumn{4}{|l|}{ Tumor number } \\
\hline$\leq 3$ & 34 & 43 & \multirow[t]{2}{*}{0.215} \\
\hline$>3$ & 52 & 45 & \\
\hline \multicolumn{4}{|l|}{ Tumor size, $\mathrm{cm}$} \\
\hline$<2$ & 12 & 28 & \multirow[t]{2}{*}{0.005} \\
\hline$\geq 2$ & 74 & 60 & \\
\hline \multicolumn{4}{|l|}{ Ascites } \\
\hline Absent & 15 & 44 & \multirow[t]{2}{*}{0.000} \\
\hline Present & 71 & 44 & \\
\hline 3-Month survival rate, \% & $25.6(22 / 86)$ & $55.7(49 / 88)$ & 0.000 \\
\hline 6-Month survival rate, $\%$ & $14.0(12 / 86)$ & $37.5(33 / 88)$ & 0.000 \\
\hline
\end{tabular}

LMR, leukocyte-to-monocyte ratio; AST, aspartate transaminase; ALP, alkaline phosphatase; BUN, blood urea nitrogen; AFU, $\alpha$-L-fucosidase; GGT, $\gamma$-glutamyl transpeptidase; PVTT, portal vein tumor thrombus; INR, international normalized ratio.

Macrophages, which are tissue-resident cells that develop from circulating monocytes in local tissues, are reported to influence tumor migration and invasion, and suppress antitumor immunity $(36,37)$. In addition, a number of studies have demonstrated platelet involvement in tumor metastasis. The activation of platelets protect tumor cells from immune elimination by promoting their arrest at the endothelium, thereby supporting the establishment of secondary lesions $(38,39)$. 
On the basis of the association between systemic inflammation and carcinoma, increasing numbers of inflammatory markers have been associated with poor prognosis of a variety of types of cancers. Chen et al (40) identified that patients with resectable gastric cancer who exhibited increased preoperative white blood cells and p-leukopenia had a poorer prognosis, compared with those with exhibited lower baseline white blood cells and no p-leukopenia. Kitayama et al (41) revealed that in patients with colorectal cancer, the lymphocyte count was markedly associated with tumor response to neoadjuvant chemoradiotherapy. Peripheral blood LMR, as a novel inflammatory biomarker, has been identified as a predictor of clinical outcome in a number of types of cancer (18-22). In regard to $\mathrm{HCC}$, to the best of our knowledge, only one study has demonstrated an association between LMR and HCC (42). Lin et al (23) identified that preoperative LMR may serve as an independent prognostic factor for patients with HBV-associated HCC undergoing curative resection. However, to the best of our knowledge, the function of LMR in HBV-associated advanced HCC remains unclear.

The present study, to the best of our knowledge, was the first to demonstrate that LMR level was independently associated with OS for patients with advanced HCC. Only patients with HBV-associated HCC who do not exhibit a fever, or any other manifestations of acute infections, were included to avoid potential confounding factors from distinct etiologies, since infection alters blood cell number. The results of the present study identified LMR, but not ALC or AMC, to be an independent predictor of the OS rate in patients with $\mathrm{HBV}$-associated advanced HCC. The aforementioned patients with an LMR $\leq 2.22$ exhibited a decreased 3 -month and 6-month survival rate compared with those with an LMR $>2.22$, which was consistent with previous studies that identified low LMR to be an independent poor prognostic factor (18-23). However, the optimal thresholds for LMR in distinct studies differ, which may be due to the different baseline characteristics, type of malignancy, clinical stages and treatments in each study.

The underlying molecular mechanism which enables LMR to be used as a prognostic factor in cancers remains unknown. Lymphocytes are a key mediator in the antitumor immunity of the host and infiltration into the tumor microenvironment is a prerequisite to an immunological antitumor reaction. ALC may alter along with the antitumor immune reaction $(35,43,44)$. ALC decreased when there was an insufficient immunological reaction to the tumor, thus promoting tumor progression and metastasis (45). Although, in the present study, ALC was not identified as a significant factor for the OS rates in patients with advanced $\mathrm{HCC}$, ALC exhibited an antitumor effect. In addition to the quantity of lyphocytes, the phenotype and subset composition may change; however, additional studies are required to determine the underlying molecular mechanism (46-48). As a component of the tumor microenvironment, monocytes serve a markedly active function in tumor development, progression and metastasis $(30,49)$. Inflammation activates the mobilization of monocytes from the bone marrow to the peripheral blood (50) and the differentiation of monocytes into tumor-associated macrophages (TAMs) following recruitment into tumor tissue $(51,52)$, suppressing adaptive immunity and exerting protumoral functions, promoting tumor cell invasion, metastasis and angiogenesis $(36,51)$. The circulating level of monocytes in the peripheral blood is reported to reflect the formation and/or presence of TAMs (45). Therefore, an increased level of monocytes reflects a high tumor burden in patients with cancer (53-55). However, in the present study, AMC was not considered a prognostic factor for the OS rate using multivariate analysis. It is hypothesized that ALC or AMC alone may not explain the effect of inflammation on patients with HBV-associated advanced HCC.

As aforementioned, LMR reflects the interaction between the immune status of the host and the tumor microenvironment. In the present study, the combination of ALC and AMC, compared with ALC and AMC alone, enabled LMR to be an improved predictor of OS rate in patients with $\mathrm{HBV}$-associated advanced $\mathrm{HCC}$, which means that decreased ALC and increased AMC simultaneously reflect the essence of the disease. Decreased LMR is associated with a poorer prognosis. A previous study demonstrated that low LMR may be considered as an independent biomarker for predicting mortality in patients with HBV-related liver cirrhosis (56), which supports the predictive value of LMR identified in the present study, as the majority of HBV-associated advanced HCC developed from liver cirrhosis. Additionally, decreased LMR was markedly associated with a number of clinicopathological characteristics, including distant metastasis, tumor size and ascites, which may reflect either higher tumor burden or a more prolonged chronic inflammatory process, indicating poor prognosis.

LMR is a readily available and low-cost objective marker of systemic inflammation and may be obtained from routine blood testing. Therefore, LMR may be used for prognostic stratification of patients with HBV-associated advanced HCC and may be a potential criterion for patient selection in clinical trials.

However, the present study had limitations. First, it is a retrospective study, the number of patients involved was small and patients were restricted to be from a single center. Secondly, LMR is a non-specific parameter of inflammation and the results may be influenced by the presence of other systemic diseases. Thirdly, the results of the present study were limited to patients with $\mathrm{HBV}$-associated advanced HCC. Additional etiologies of HCC, including $\mathrm{HCV}$ and alcohol, require study. Finally, the heterogeneity of patients with advanced HCC includes the distinctions between metastatic sites, which may bias the results. Additional large-scale prospective studies and standard investigations are required to validate the results of the present study.

The present study, to the best of our knowledge, was the first study to analyze the prognosis of patients with HBV-associated advanced HCC on the basis of a novel biomarker, LMR. LMR was identified to be an independent prognostic factor of patients with $\mathrm{HBV}$-associated advanced HCC with increased baseline LMR levels indicating an improved prognosis. A prospective and well-designed study of LMR with larger cohorts is warranted for further validation.

\section{Acknowledgements}

The present study was supported by the National Natural Science Foundation of China (grant nos. 31600710 and 81372374), the Natural Science Foundation of Guangdong (grant nos. 2014A030313146 and 2016A030313302) and Project 
on the Integration of Industry, Education and Research of Guangdong Province (grant no. 2012B091100460)

\section{References}

1. Siegel RL, Miller KD and Jemal A: Cancer statistics, 2015. CA Cancer J Clin 65: 5-29, 2015.

2. Chen W, Zheng R, Baade PD, Zhang S, Zeng H, Bray F, Jemal A, Yu XQ and He J: Cancer statistics in China, 2015. CA Cancer J Clin 66: 115-132, 2016.

3. Yuan JM, Govindarajan S, Arakawa K and Yu MC: Synergism of alcohol, diabetes and viral hepatitis on the risk of hepatocellular carcinoma in blacks and whites in the U.S. Cancer 101: 1009-1017, 2014

4. Bruix J and Sherman M; Practice Guidelines Committee, American Association for the Study of Liver Diseases. Management of hepatocellular carcinoma. Hepatology 42: 1208-1236, 2005.

5. Li J, Wang L, Cong N, Shi C, Bu W, Song J and Chen H: Efficacy of sorafenib for advanced hepatocellular carcinoma and prognostic factors. Hepatogastroenterology 61: 954-957, 2014.

6. Ogasawara S, Chiba T, Ooka Y, Suzuki E, Kanogawa N, Saito T, Motoyama T, Tawada A, Kanai F and Yokosuka O: Post-progression survival in patients with advanced hepatocellular carcinoma resistant to sorafenib. Invest New Drugs 34: 255-260, 2016.

7. Hong YF, Chen ZH, Ma XK, Li X, Wu DH, Chen J, Dong M, Wei L, Wang TT, Ruan DY, et al: Comparison of five models for end-stage liver disease in predicting the survival rate of patients with advanced hepatocellular carcinoma. Tumour Biol 37 : 5265-5273, 2015 .

8. Sherman M: Hepatocellular carcinoma: Epidemiology, surveillance, and diagnosis. Semin Liver Dis 30: 3-16, 2010.

9. Bugada D, Allegri M, Lavand'homme P, De Kock M and Fanelli G: Inflammation-based scores: A new method for patient-targeted strategies and improved perioperative outcome in cancer patients. Biomed Res Int 2014: 142425, 2014.

10. Deng Q, He B, Liu X, Yue J, Ying H, Pan Y, Sun H, Chen J, Wang F, Gao T, et al: Prognostic value of pre-operative inflammatory response biomarkers in gastric cancer patients and the construction of a predictive model. J Transl Med 13: 66, 2015.

11. Shiels MS, Katki HA, Hildesheim A, Pfeiffer RM, Engels EA, Williams M, Kemp TJ, Caporaso NE, Pinto LA and Chaturvedi AK: Circulating inflammation markers, risk of lung cancer, and utility for risk stratification. J Natl Cancer Inst 107: djv199, 2015

12. Morrison L, Laukkanen JA, Ronkainen K, Kurl S, Kauhanen J and Toriola AT: Inflammatory biomarker score and cancer: A population-based prospective cohort study. BMC Cancer 16: 80, 2016.

13. Fang S, Wang Y, Sui D, Liu H, Ross MI, Gershenwald JE, Cormier JN, Royal RE, Lucci A, Schacherer CW, et al: C-reactive protein as a marker of melanoma progression. J Clin Oncol 33: 1389-1396, 2015

14. Aleksandrova K, Boeing H, Nöthlings U, Jenab M, Fedirko V, Kaaks R, Lukanova A, Trichopoulou A, Trichopoulos D, Boffetta P, et al: Inflammatory and metabolic biomarkers and risk of liver and biliary tract cancer. Hepatology 60: 858-871, 2014.

15. Schumacher D, Strilic B, Sivaraj KK, Wettschureck N and Offermanns S: Platelet-derived nucleotides promote tumor-cell transendothelialmigration and metastasis via $\mathrm{p} 2 \mathrm{y} 2$ receptor. Cancer Cell 24: 130-137, 2013

16. Grosse-Steffen T, Giese T, Giese N, Longerich T, Schirmacher P, Hansch GM and Gaida MM: Epithelial-to-mesenchymal transition in pancreatic ductal adenocarcinoma and pancreatic tumor cell lines: The role of neutrophils and neutrophil-derived elastase. Clin Dev Immunol 2012: 720768, 2012.

17. Ren QQ, Fu SJ, Zhao Q, Guo ZY, Ji F, Chen MG, Wu LW and He XS: Prognostic value of preoperative peripheral monocyte count in patients with hepatocellular carcinoma after liver transplantation. Tumor Biol 37: 8973-8988, 2016.

18. Jia W, Wu J, Jia H, Yang Y, Zhang X, Chen K and Su F: The peripheral blood Neutrophil-to-Lymphocyte ratio is superior to the Lymphocyte-to-Monocyte ratio for predicting the long-term survival of triple-negative breast cancer patients. PLoS One 10 e0143061, 2015.

19. Chang Y, Fu Q, Xu L, Zhou L, Liu Z, Yang Y, Lin Z and Xu J: Prognostic value of preoperative lymphocyte to monocyte ratio in patients with nonmetastatic clear cell renal cell carcinoma. Tumor Biol 37: 4613-4620, 2016
20. Chang Y, An H, Xu L, Zhu Y, Yang Y, Lin Z and Xu J: Systemic inflammation score predicts postoperative prognosis of patients with clear-cell renal cell carcinoma. Br J Cancer 113: 626-633, 2015.

21. Chen YM, Lai CH, Chang HC, Chao TY, Tseng CC, Fang WF, Wang CC, Chung YH, Wang YH, Su MC, et al: Baseline and trend of Lymphocyte-to-Monocyte ratio as prognostic factors in epidermal growth factor receptor mutant non-small cell lung cancer patients treated with first-line epidermal growth factor tyrosine kinase inhibitors. PLoS One 10: e0136252, 2015.

22. Shibutani M, Maeda K, Nagahara H, Ohtani H, Sakurai K, Yamazoe S, Kimura K, Toyokawa T, Amano R, Tanaka H, et al: Prognostic significance of the lymphocyte-to-monocyte ratio in patients with metastatic colorectal cancer. World J Gastroenterol 21: 9966-9973, 2015.

23. Lin ZX, Ruan DY, Li Y, Wu DH, Ma XK, Chen J, Chen ZH, Li X, Wang TT, Lin Q, et al: Lymphocyte-to-monocyte ratio predicts survival of patients with hepatocellular carcinoma after curative resection. World J Gastroenterol 21: 10898-10906, 2015.

24. Llovet JM, Brú C and Bruix J: Prognosis of hepatocellular carcinoma: The BCLC staging classification. Semin Liver Dis 19: 329-338, 1999.

25. National Comprehensive Cancer Network. NCCN Guidelines Version 2.2105 Hepatocellular Carcinoma.

26. Schag CC, Heinrich RL and Ganz PA: Karnofsky performance status revisited: Reliability, validity, and guidelines. J Clin Oncol 2: 187-193, 1984.

27. Fluss R, Faraggi D and Reiser B: Estimation of the Youden Index and its associated cutoff point. Biom J 47: 458-472, 2005.

28. Keibel A, Singh V and Sharma MC: Inflammation, microenvironment, and the immune system in cancer progression. Curr Pharm Des 15: 1949-1955, 2009.

29. Balkwill $\mathrm{F}$ and Mantovani A: Inflammation and cancer: Back to Virchow? Lancet 357: 539-545, 2001.

30. Mantovani A, Allavena P, Sica A and Balkwill F: Cancer-related inflammation. Nature 454: 436-444, 2008.

31. Wang Y, Niu XL, Qu Y, Wu J, Zhu YQ, Sun WJ and Li LZ: Autocrine production of interleukin- 6 confers cisplatin and paclitaxel resistance in ovarian cancer cells. Cancer Lett 295: $110-123,2015$.

32. Kuang DM, Zhao Q, Wu Y, Peng C, Wang J, Xu Z, Yin XY and Zheng L: Peritumoral neutrophils link inflammatory response to disease progression by fostering angiogenesis in hepatocellular carcinoma. J Hepatol 54: 948-955, 2011.

33. Lee IK, Vansaun MN, Shim JH, Matrisian LM and Gorden DL: Increased metastases are associated with inflammation and matrix metalloproteinase- 9 activity at incision sites in a murine model of peritoneal dissemination of colorectal cancer. J Surg Res 180: 252-259, 2013.

34. Mishalian I, Granot $Z$ and Fridlender ZG: The diversity of circulating neutrophils in cancer. Immunobiology 222: 82-88, 2017.

35. Dunn GP, Old LJ and Schreiber RD: The immunobiology of cancer immunosurveillance and immunoediting. Immunity 21 : 137-148, 2004.

36. Condeelis J and Pollard JW: Macrophages: Obligate partners for tumor cell migration, invasion, and metastasis. Cell 124: 263-266, 2006

37. Leek RD and Harris AL: Tumor-associated macrophages in breast cancer. J Mammary Gland Biol Neoplasia 7: 177-189, 2002.

38. Gay LJ and Felding-Habermann B: Contribution of platelets to tumour metastasis. Nat Rev Cancer 11: 123-134, 2011.

39. Buergy D, Wenz F, Groden C and Brockmann MA: Tumor-platelet interaction in solid tumors. Int J Cancer 130: 2747-2760, 2012.

40. Chen XF, Qian J, Pei D, Zhou C, Røe OD, Zhu F, He SH, Qian YY, Zhou Y, Xu J, et al: Prognostic value of perioperative leukocyte count in resectable gastric cancer. World J Gastroenterol 22: 2818-2827, 2016.

41. Kitayama J, Yasuda K, Kawai K, Sunami E and Nagawa H: Circulating lymphocyte number has a positive association with tumor response in neoadjuvant chemoradiotherapy for advanced rectal cancer. Radiat Oncol 5: 47, 2010.

42. Gu L, Li H, Chen L, Ma X, Li X, Gao Y, Zhang Y, Xie Y and Zhang X: Prognostic role of lymphocyte to monocyte ratio for patients with cancer: Evidence from a systematic review and meta-analysis. Oncotarget 7: 31926-31942, 2016.

43. Hoffmann TK, Dworacki G, Tsukihiro T, Meidenbauer N, Gooding W, Johnson JT and Whiteside TL: Spontaneous apoptosis of circulating $\mathrm{T}$ lymphocytes in patients with head and neck cancer and its clinical importance. Clin Cancer Res 8: 2553-2562, 2002. 
44. Rabinowich H, Cohen R, Bruderman I, Steiner Z and Klajman A: Functional analysis of mononuclear cells infiltrating into tumors: Lysis of autologous human tumor cells by cultured infiltrating lymphocytes. Cancer Res 47: 173-177, 1987.

45. Stotz M, Pichler M, Absenger G, Szkandera J, Arminger F, Schaberl-Moser R, Samonigg H, Stojakovic T and Gerger A: The preoperative lymphocyte to monocyte ratio predicts clinical outcome in patients with stage III colon cancer. Br J Cancer 110 435-440, 2014

46. Unitt E, Marshall A, Gelson W, Rushbrook SM, Davies S, Vowler SL, Morris LS, Coleman N and Alexander GJ: Tumour lymphocytic infiltrate and recurrence of hepatocellular carcinoma following liver transplantation. J Hepatol 45: 246-253, 2006.

47. Zikos TA, Donnenberg AD, Landreneau RJ, Luketich JD and Donnenberg VS: Lung T-cell subset composition at the time of surgical resection is a prognostic indicator in nonsmall cell lung cancer. Cancer Immunol Immunother 60: 819-827, 2011.

48. Gao Q, Qiu SJ, Fan J, Zhou J, Wang XY, Xiao YS, Xu Y, Li YW and Tang ZY: Intratumoral balance of regulatory and cytotoxic $\mathrm{T}$ cells is associated with prognosis of hepatocellular carcinoma after resection. J Clin Oncol 25: 2586-2593, 2007.

49. Evani SJ, Prabhu RG, Gnanaruban V, Finol EA and Ramasubramanian AK: Monocytes mediate metastatic breast tumor cell adhesion to endothelium under flow. FASEB J 27 $3017-3029,2013$
50. Shi C and Pamer EG: Monocyte recruitment during infection and inflammation. Nat Rev Immunol 11: 762-774, 2011.

51. Pollard JW: Tumour-educated macrophages promote tumour progression and metastasis. Nat Rev Cancer 4: 71-78, 2004.

52. Hagemann $\mathrm{T}$ and Lawrence $\mathrm{T}$ : Investigating macrophage and malign interactions in vitro. Methods Mol Biol 512: 325-332, 2009.

53. Tadmor T: Does monocyte count have prognostic significance in cancer? Leuk Res 37: 1193-1194, 2013.

54. Sasaki A, Iwashita Y, Shibata K, Matsumoto T, Ohta M and Kitano S: Prognostic value of preoperative peripheral blood monocyte count inpatients with hepatocellular carcinoma. Surgery 139: 755-764, 2006.

55. Lin GN, Jiang XM, Peng JW, Xiao JJ, Liu DY and Xia ZJ: Prognostic significance of the peripheral blood absolute monocyte count inpatients with locally advanced or metastatic hepatocellular carcinoma receiving systemic chemotherapy. Asian Pac J Cancer Prev 15: 6387-6390, 2014.

56. Zhang J, Feng G, Zhao Y, Zhang J, Feng L and Yang J: Association between lymphocyte-to-monocyte ratio (LMR) and the mortality of HBV-related liver cirrhosis: A retrospective cohort study. BMJ Open 5: e008033, 2015. 